\title{
Avaliação de pacientes hipertensos acompanhados pelo Programa Saúde da Família em um Centro de Saúde Escola*
}

\author{
Evaluation from the hypertensive patients followed in the Family Health Program \\ at Health School Center \\ Evaluación de los pacientes hipertensos acompañado del Programa Salud de \\ Familia del Centro de Salud Escuela
}

\author{
Gisele Machado Peixoto Mano', Angela Maria Geraldo Pierin²
}

\begin{abstract}
RESUMO
Objetivo: Comparar dois grupos de hipertensos: um atendido no programa tradicional e outro acompanhado pelo Programa Saúde da Família (PSF). Método: Foram analisados 113 prontuários de hipertensos do programa tradicional e 113 do PSF. Resultados: Os hipertensos do PSF foram estatisticamente diferentes dos demais $(p<0,05)$ nos seguintes aspectos: raça; grau de escolaridade; ocupação; idade; peso; número de refeições ao dia; ingestão de café; tabagismo; ingestão de bebida alcoólica; atividade física; tempo de diagnóstico de hipertensão; antecedentes familiares para a doença; tipos de doenças associadas; exames laboratoriais; tipos de atendimentos e participação em grupos específicos. Os hipertensos do PSF apresentaram ainda, queda significativa da pressão arterial (152 $\pm 24 / 92 \pm 15$ vs $142 \pm 21 / 85 \pm 13 \mathrm{~mm} \mathrm{Hg}$ ) entre o início e o final do período de estudo, com pressão sistólica inicial maior que o programa tradicional. Apenas $30 \%$ dos hipertensos do programa tradicional e $20 \%$ do PSF estavam com a pressão arterial controlada. Conclusão: Apesar do controle pouco satisfatório da pressão os hipertensos seguidos pelo PSF apresentaram redução significativa dos níveis pressóricos.
\end{abstract}

Descritores: Hipertensão; Programa Saúde da Família

\section{ABSTRACT}

Objective: The study compared two groups of hypertensive patients: one attended by the traditional process of basic health unit care and the other one followed by the Family Health Program (FHP). Method: 113 records of hypertensive patients attended by the traditional process basic heath unit care and 113 by the FHP Results: The results showed that hypertensive patients from FHP were different $(p<0.05)$ in relation to the others on the predominance of the white race; lower education level; fewer workers; older age and higher weight; more tobacco users; less alcoholic beverage, less physical activity, and longer time of hypertension. The patients from FHP had more nursing appointments, nursing home visit, medical home visit, and participation in specific groups; however, there were fewer medical appointments and more attendance at the emergence room. The patients at FHP presented a significant reduction in the blood pressure $(152 \pm 24 / 92 \pm 15$ vs $142 \pm 21 / 85 \pm 13 \mathrm{~mm} \mathrm{Hg})$ from the beginning to the end of the period of the study with initial systolic blood pressure higher than the patients from the traditional program. Conclusion: Only $30 \%$ of the patients from the traditional program and $20 \%$ from FHP had their blood pressure controlled.

Keywords: Hypertension; Family Health Program

\section{RESUMEN}

Objetivo: Este trabajo compara dos grupos de hipertensos: uno atendido por el proceso tradicional de asistencia en la unidad básica de salud y otro acompañado por el programa salud de la familia (PSF). Método: Fueran analizados 113 prontuarios de cada grupo Resultados: Los hipertensos del PSF fueron diferentes $(p<0,05)$ con relación a los demás, con predominio en la raza blanca; menor escolaridad; menos trabajadores; edad y peso más elevados; menos bebida alcohólica; menos actividad física; más tiempo de diagnóstico de hipertensión; y menos antecedentes familiares para la enfermedad. Los hipertensos del PSF tuvieron más consultas de enfermería, visita domiciliaria de enfermería, visita domiciliaria médica, participación en grupos específicos, sin embargo hubo menos consultas médicas y más situaciones de atendimiento rápido. Los hipertensos del PSF presentaron una baja significativa de la presión arterial entre el inicio y el final del período de estudio (152 $\pm 24 / 92 \pm 15$ vs. $142 \pm 21 / 85 \pm 13 \mathrm{~mm} \mathrm{Hg}$ ) con presión sistólica inicial mayor de que los hipertensos del programa tradicional. Conclusión: Apenas 30\% de los hipertensos del programa tradicional y $20 \%$ del PSF estaban con la presión arterial controlada.

Descriptores: Hipertensión; Programa Salud de la Familia

Trabalho extraído da dissertação de Mestrado apresentada ao Programa de Pós Graduação - Enfermagem da Saúde do Adulto, da Escola de Enfermagem da Universidade de São Paulo - USP - São Paulo (SP), Brasil.

1 Enfermeira assistencial no Programa Saúde da Família do Centro de Saúde Escola Barra Funda Dr Alexandre Vranjac, São Paulo $(S P)$, Brasil.

2 Professora Livre-Docente da Escola de Enfermagem da Universidade de São Paulo. 


\section{INTRODUÇÃO}

Atualmente, sabe-se que tanto nos países desenvolvidos quanto nos países em desenvolvimento, predomina a mortalidade por doença cardiovascular ${ }^{(1)}$. Como causa isolada, a hipertensão arterial é a mais importante morbidade do adulto. Mesmo face à efetividade do tratamento medicamentoso e não medicamentoso, é uma doença de difícil controle provavelmente devido à baixa adesão ao tratamento. Os profissionais da área da saúde devem buscar estratégias para melhorar a adesão dos hipertensos ao tratamento. Nesse sentido, o Programa Saúde da Família pode ser útil pois, pode ajudar os hipertensos a obterem o controle da doença no contexto da família.

No Brasil, estima-se que haja um número elevado de pessoas adultas com hipertensão arterial em tratamento nas instituições de saúde. Não há levantamentos de prevalência de hipertensão arterial como um todo, porém estudos isolados mostram variações de $22,3 \%$ a $43,9 \%(2-5)$.

Muitos fatores ${ }^{(6-7)}$ predispõem os indivíduos ao aumento da pressão arterial. A herança genética é o único que não é modificável. Os demais, como a ingestão de muito sal, estresse, obesidade, sedentarismo e ingestão de bebidas alcoólicas em excesso são passíveis de modificação. Portanto, a atuação dos profissionais de saúde no controle da hipertensão deve prever a adoção de hábitos de vida saudáveis.

O controle da hipertensão arterial é pouco satisfatório, estudos em nosso meio mostram que apenas cerca de $30 \%$ dos hipertensos estão controlados ${ }^{(8-10)}$.

Considera-se que os baixos níveis de controle da doença tenham relação direta com a pouca adesão ao tratamento. Promover adesão ao tratamento da hipertensão arterial, por meio de estratégias que elevem o controle da doença traz benefícios não só para as instituições de saúde, bem como melhoram o tratamento nesse nível de intervenção. Desta forma disponibilizam vagas nas instituições terciárias, reduzindo o número de acidente vascular encefálico, a ocorrência de insuficiência cardíaca congestiva, doença renal e doença arterial coronária, que são complicações decorrentes do controle inadequado da hipertensão arterial.

O Programa Saúde da Família é uma estratégia que representa um dos principais eixos de ação do Ministério da Saúde para mudar o modelo de assistência à saúde no país. A estratégia prioriza as ações de promoção, proteção e recuperação da saúde dos indivíduos e da família, do recém nascido ao idoso, sadios ou doentes, de forma integral e/ou contínua. Com base em manuais editados pelo Ministério da Saúde ${ }^{(11)}$, o programa oferece grande ajuda na detecção precoce da hipertensão arterial realizando medidas regulares da pressão arterial, assim como, prevenindo e controlando outras doenças crônicas. Com o acesso dos profissionais de saúde às famílias de hipertensos há o favorecimento nos esforços de prevenção, incentivando tanto os portadores da doença como a seus familiares a adotarem hábitos de vida saudáveis e controlando, corrigindo e evitando maiores complicações ${ }^{(12)}$.

Assim, reconhecendo a importância do trabalho em conjunto da equipe de saúde e a necessidade de se conhecer a influência das atividades do Programa Saúde da Família no controle dos hipertensos em atendimento em uma Unidade Básica de Saúde o presente estudo teve como objetivo, caracterizar um grupo de pacientes hipertensos seguidos no Programa Saúde da Família em um Centro de Saúde Escola.

\section{MÉTODO}

Trata-se de um estudo com abordagem quantitativa, não - experimental, do tipo survey exploratório, de campo e transversal, realizado por meio de consulta aos prontuários dos pacientes.

O estudo foi realizado em um Centro de Saúde Escola, do Município de São Paulo, que atende moradores e trabalhadores de empresas de pequeno porte na região da Barra Funda. O serviço desenvolve o Programa Saúde da Família (PSF) como de estratégia de atendimento. O sistema de atendimento tradicional ainda permanecia em vigor por ocasião da coleta de dados. O Centro de Saúde Escola existe há 35 anos e no momento do estudo possuía 17.465 pessoas matriculadas.

A casuística foi calculada para um nível de significância de 0,05 e um poder de 0,80 , totalizando 226 prontuários, sendo 113 de hipertensos seguidos pelo PSF e 113 pelo sistema tradicional. Os pacientes dos dois grupos foram sorteados aleatoriamente. Os critérios de inclusão de pacientes no estudo foram: possuir hipertensão arterial primária, estar em acompanhamento no período de estudo, idade maior ou igual a 18 anos e não ser paciente obstétrico. O projeto foi aprovado pelo Comitê de Ética da Faculdade de Ciências Médicas da Irmandade Santa Casa de Misericórdia de São Paulo.

As variáveis qualitativas foram analisadas com o teste qui-quadrado ou teste da razão de verossimilhança. As variáveis quantitativas são apresentadas em tabelas contendo médias e desvios padrão e foram avaliadas com o teste t-Student e teste não paramétrico da soma de postos de Wilcoxon.

Para as pressões, mensuradas em mais de uma condição de avaliação (inicial e final), foi utilizada a técnica de análise de variância para medidas repetidas ${ }^{(13)}$.

Os valores de $\mathrm{p}<0,05$ foram considerados estatisticamente significantes. 
Os dados coletados nos prontuários incluíram aspectos biossociais; hábitos de vida como alimentação, atividade física, fumo e bebida alcoólica; histórico da doença; tratamento com e sem medicamentos; participação em consultas e grupos; resultados de exames como perfil glicêmico, lipídico e urina I; doenças associadas; e caracterização de níveis de pressão arterial por data e tipo de consulta.

\section{RESULTADOS}

Os dados da Tabela 1 mostram as características dos pacientes estudados com predominância do sexo feminino (63\%), idade de 58,5 $\pm 13,5$ anos, de cor branca (67\%), brasileiros $(56 \%)$, com ensino fundamental completo $(40 \%)$, trabalhadores (49\%) e com renda entre um e três salários mínimos salário mínimo (57\%). Quanto aos hábitos de vida (Tabela 2), $18 \%$ eram tabagistas, $21 \%$ etilistas e $26 \%$ realizavam atividade física somente em casa e no trabalho mais que 30 minutos e mais que três vezes na semana. Nos hábitos alimentares, uma pequena parcela apontou dieta com diminuição de sal (5\%). Informa-se ainda, que pouco mais da metade $(56 \%)$ realizava três refeições ao dia.

Tabela 1 - Características biossociais dos hipertensos atendidos no Programa Tradicional e no Programa de Saúde da Família (São Paulo, 2004)

\begin{tabular}{|c|c|c|c|c|c|c|c|c|c|c|c|c|}
\hline \multirow{3}{*}{ Características } & \multicolumn{4}{|c|}{ Programa Tradicional } & \multicolumn{4}{|c|}{ Programa Saúde da Família } & \multicolumn{4}{|c|}{ Total } \\
\hline & \multicolumn{2}{|c|}{ Consta } & \multicolumn{2}{|c|}{$\begin{array}{c}\text { Não } \\
\text { Consta }\end{array}$} & \multicolumn{2}{|c|}{ Consta } & \multicolumn{2}{|c|}{$\begin{array}{c}\text { Não } \\
\text { Consta }\end{array}$} & \multicolumn{2}{|c|}{ Consta } & \multicolumn{2}{|c|}{$\begin{array}{l}\text { Não } \\
\text { Consta }\end{array}$} \\
\hline & $\mathbf{N}$ & $\%$ & $\mathbf{N}$ & $\%$ & $\mathbf{N}$ & $\%$ & $\mathbf{N}$ & $\%$ & $\mathbf{N}$ & $\%$ & $\mathbf{N}$ & $\%$ \\
\hline Sexo & 113 & 100 & - & & 113 & 100 & - & & 226 & 100 & - & 0 \\
\hline Feminino & 79 & 70,0 & & & 63 & 56,0 & & & 142 & 63,0 & & \\
\hline Masculino & 34 & 30,0 & & & 50 & 44,0 & & & 84 & 37,0 & & \\
\hline Etnia* & 105 & 93 & 8 & & 113 & 100 & - & & 218 & 96,5 & 8 & 7,0 \\
\hline Branca & 58 & 55,0 & & & 88 & 78,0 & & & 146 & 67,0 & & \\
\hline Não branco & 47 & 45,0 & & & 25 & 22,0 & & & 72 & 33,0 & & \\
\hline Nacionalidade & 113 & 100 & - & & 113 & 100 & - & & 226 & 100 & - & 0 \\
\hline Brasileiro & 72 & 64,0 & & & 55 & 49,0 & & & 127 & 56,0 & & \\
\hline Estrangeiro & 41 & 36,0 & & & 58 & 51,0 & & & 99 & 44,0 & & \\
\hline $\begin{array}{l}\text { Renda (salários } \\
\text { mínimos) }\end{array}$ & 94 & 83 & 19 & 17 & 83 & 73 & 30 & 26,5 & 177 & 76 & 49 & 22,0 \\
\hline Sem rendimento & 42 & 45,0 & & & 25 & 30,0 & & & 67 & 38,0 & & \\
\hline $1-3$ & 50 & 53,0 & & & 51 & 62,0 & & & 101 & 57,0 & & \\
\hline$\geq 4$ & 2 & 2,0 & & & 7 & 8,0 & & & 9 & 5,0 & & \\
\hline Ocupação* & 105 & 93 & 8 & 7 & 87 & 77 & 26 & 23 & 192 & 85 & 34 & 15,0 \\
\hline Aposentados/ & 33 & 31,0 & & & 49 & 56,0 & & & 82 & 43,0 & & \\
\hline Trabalhadores & 72 & 69,0 & & & 38 & 44,0 & & & 110 & 57,0 & & \\
\hline $\begin{array}{l}\text { Grau de } \\
\text { instrução* }\end{array}$ & 113 & 100 & - & 0 & 112 & 99 & 1 & 1 & 225 & 99,5 & 1 & 0,5 \\
\hline Não informado/ & - & - & & & 6 & 5,0 & & & 6 & 3,0 & & \\
\hline $\begin{array}{l}\text { Analfabeto, } \\
\text { sabe ler/escrever }\end{array}$ & 10 & 9,0 & & & 22 & 20,0 & & & 32 & 14,0 & & \\
\hline $\begin{array}{l}\text { Fundamental } \\
\text { incompleto }\end{array}$ & 19 & 17,0 & & & 29 & 26,0 & & & 48 & 21,0 & & \\
\hline completo & 65 & 57,5 & & & 26 & 23,0 & & & 91 & 40,0 & & \\
\hline Médio & 4 & 3,5 & & & 4 & 4,0 & & & 8 & 4,0 & & \\
\hline completo & 13 & 11,0 & & & 11 & 10,0 & & & 24 & 11,0 & & \\
\hline Superior & 1 & 1,0 & & & 9 & 8,0 & & & 10 & 4,0 & & \\
\hline completo & 1 & 1,0 & & & 5 & 4,0 & & & 6 & 3,0 & & \\
\hline Idade* & 112 & 99 & 1 & 1 & 112 & 99 & 1 & 1 & 224 & 99 & 2 & 1,0 \\
\hline $\begin{array}{l}\text { Anos (média } \pm \\
\text { DP) }\end{array}$ & $55 \pm 12$ & & & & $62 \pm 15$ & & & & $58,5 \pm 13,5$ & & & \\
\hline $\begin{array}{l}\text { Peso* } \\
\text { Kg (média } \pm \text { DP) }\end{array}$ & $\begin{array}{c}107 \\
70 \pm 11\end{array}$ & 95 & 6 & 5 & $\begin{array}{c}88 \\
77 \pm 20\end{array}$ & 78 & 25 & 22 & $\begin{array}{c}195 \\
73,5 \pm 15,5\end{array}$ & 86,0 & 31 & 14,0 \\
\hline Altura & 110 & 97 & 3 & 3 & 99 & 88 & 14 & 12 & 209 & 92,5 & 4 & 2,0 \\
\hline $\begin{array}{l}M \text { (média } \pm \mathrm{DP}) \\
\text { Índice de massa }\end{array}$ & $1,59 \pm 0,08$ & & & & $1,60 \pm 0,09$ & & & & $1,60 \pm 0,09$ & & & \\
\hline $\begin{array}{l}\text { corporal } \mathrm{Kg} / \mathrm{m}^{2} \\
\text { (média } \pm \mathrm{DP} \text { ) }\end{array}$ & $28 \pm 3,95$ & & & & $29 \pm 6,55$ & & & & $28,5 \pm 5$ & & & \\
\hline
\end{tabular}

$$
{ }^{*} p<0,05
$$


Tabela 2 - Atividade física, tabagismo, ingestão de bebida alcoólica e hábitos alimentares dos hipertensos atendidos no Programa Tradicional e no Programa de Saúde da Família (São Paulo, 2004)

\begin{tabular}{|c|c|c|c|c|c|c|c|c|c|c|c|c|}
\hline \multirow{3}{*}{ Características } & \multicolumn{4}{|c|}{ Programa Tradicional } & \multicolumn{4}{|c|}{$\begin{array}{l}\text { Programa Saúde } \\
\text { da Família }\end{array}$} & \multicolumn{4}{|c|}{ Total } \\
\hline & \multicolumn{2}{|c|}{ Consta } & \multicolumn{2}{|c|}{$\begin{array}{l}\text { Não } \\
\text { consta }\end{array}$} & \multicolumn{2}{|c|}{ Consta } & \multicolumn{2}{|c|}{$\begin{array}{l}\text { Não } \\
\text { consta }\end{array}$} & \multicolumn{2}{|c|}{ Consta } & \multicolumn{2}{|c|}{$\begin{array}{l}\text { Não } \\
\text { consta }\end{array}$} \\
\hline & $\mathbf{N}$ & $\%$ & $\mathbf{N}$ & $\%$ & $\mathbf{N}$ & $\%$ & $\mathbf{N}$ & $\%$ & $\mathbf{N}$ & $\%$ & $\mathbf{N}$ & $\%$ \\
\hline Atividade física* & 102 & 90,0 & 11 & 10,0 & 78 & 69,0 & 35 & 31,0 & 180 & 80,0 & 46 & 20,0 \\
\hline Não & 22 & 22,0 & & & 38 & 49,0 & & & 60 & 33,0 & & \\
\hline $\begin{array}{l}\text { Só em casa ou } \\
\text { trabalho }\end{array}$ & 25 & 24,0 & & & 22 & 28,0 & & & 47 & 26,0 & & \\
\hline $\operatorname{Sim}$ & 55 & 54,0 & & & 18 & 23,0 & & & 73 & 40,0 & & \\
\hline Tabagismo* & 92 & 81,0 & 21 & 19,0 & 60 & 53,0 & 53 & 47,0 & 152 & 67,0 & 74 & 33,0 \\
\hline Não & 77 & 84,0 & & & 47 & 78,0 & & & 124 & 81,0 & & \\
\hline Sim & 15 & 16,0 & & & 13 & 22,0 & & & 28 & 18,0 & & \\
\hline Bebida alcoólica* & 61 & 54,0 & 52 & 46,0 & 41 & 36,0 & 72 & 64,0 & 102 & 45,0 & 124 & 55,0 \\
\hline Não & 46 & 75,0 & & & 35 & 85,0 & & & 81 & 79,0 & & \\
\hline Sim & 15 & 25,0 & & & 6 & 15,0 & & & 21 & 21,0 & & \\
\hline Hábitos alimentares & 49 & 43,0 & 64 & 57,0 & 53 & 47,0 & 60 & 53,0 & 102 & 45,0 & 124 & 55,0 \\
\hline Diminuiu sal & 2 & 2,0 & & & 3 & 3,0 & & & 5 & 5,0 & & \\
\hline Restrição de gordura & 26 & 23,0 & & & 22 & 20,0 & & & 48 & 47,0 & & \\
\hline Legumes/frutas/ & & & & & & & & & & & & \\
\hline verduras & 44 & 39,0 & & & 34 & 30,0 & & & 78 & 76,5 & & \\
\hline Ingestão de café* & 2 & 2,0 & & & 22 & 19,5 & & & 24 & 23,5 & & \\
\hline
\end{tabular}

Os hipertensos seguidos pelo PSF foram significativamente diferentes $(\mathrm{p}<0,05)$ dos pacientes atendidos pelo sistema tradicional em relação a: etnia, com predomínio de brancos (78\% vs 55\%); grau de escolaridade, apenas $23 \%$ tinham ensino fundamental completo; menos trabalhadores (44\% vs 69\%); idade mais elevada (62 \pm 15 vs $55 \pm 12$ anos); peso corporal mais elevado $(77 \pm 20$ vs $70 \pm 11 \mathrm{Kg})$; fazem menos refeições $(3$ refeições ao dia, $8 \%$ vs $23 \%$ ); bebem mais café $(19,5 \%$ vs $2 \%$ ); fumam mais ( $22 \%$ vs $16 \%$ ); ingerem menos bebida alcoólica (15\% vs 25\%); fazem menos atividade física (14\% vs 39\%); maior tempo de diagnóstico de hipertensão (16\% vs 12\%); e menos antecedentes familiares para hipertensão (11\% vs 26\%). Com relação às doenças associadas tem menos obesidade $(20 \% v s$ $36 \%)$, mais doenças músculoesqueléticas (15\% vs 7\%) e do aparelho circulatório (32\% vs $18 \%$ ). Nos exames laboratoriais, apresentaram níveis de triglicérides séricos mais elevados ( $\mathrm{p}<0,05,175 \pm 119$ vs $136 \pm 68 \mathrm{mg} / \mathrm{dl}$ ).

Os pacientes seguidos pelo programa tradicional em relação aos hipertensos do PSF foram mais atendidos $(\mathrm{p}<0,05)$ por meio de consulta médica $(96,5 \%$ vs $86 \%)$ e atendimentos pelo auxiliar de enfermagem ( $41 \%$ vs $8 \%)$. As outras formas de atendimento foram mais freqüentes $(p<0,05)$ nos hipertensos do PSF e incluíram: consultas com enfermeira, visita domiciliar médica, visita domiciliar de enfermagem, participação nos grupos de qualidade de vida, Lian Gong (ginástica oriental) e caminhada. (Tabela 3)

Tabela 3 - Participação nos atendimentos e grupos oferecidos aos hipertensos atendidos no Programa de Saúde da Família e no Programa Tradicional (São Paulo, 2004)

\begin{tabular}{|c|c|c|c|c|c|c|}
\hline \multirow[b]{2}{*}{ Atendimento e Grupos } & \multicolumn{2}{|c|}{$\begin{array}{c}\text { Programa Saúde da } \\
\text { Família }\end{array}$} & \multicolumn{2}{|c|}{$\begin{array}{c}\text { Programa } \\
\text { Tradicional }\end{array}$} & \multicolumn{2}{|c|}{ Total } \\
\hline & $\mathbf{N}$ & $\%$ & $\mathbf{N}$ & $\%$ & $\mathbf{N}$ & $\%$ \\
\hline Consulta Médica* & 97 & 86,0 & 109 & 96,5 & 206 & 91,0 \\
\hline Pronto atendimento & 50 & 44,0 & 54 & 48,0 & 104 & 46,0 \\
\hline $\begin{array}{l}\text { Consulta de Auxiliar de } \\
\text { enfermagem }{ }^{*}\end{array}$ & 9 & 8,0 & 46 & 41,0 & 55 & 24,0 \\
\hline Consulta de enfermeira* & 34 & 30,0 & - & - & 34 & 15,0 \\
\hline $\begin{array}{l}\text { Visita domiciliar de } \\
\text { enfermagem* }\end{array}$ & 25 & 22,0 & 1 & 1,0 & 26 & 11,5 \\
\hline Visita domiciliar médica* & 21 & 19,0 & 3 & 3,0 & 24 & 11,0 \\
\hline $\begin{array}{l}\text { Grupo medida de pressão } \\
\text { arterial }\end{array}$ & 8 & 7,0 & 5 & 4,0 & 13 & 6,0 \\
\hline Grupo de qualidade de vida* & 6 & 5,0 & - & - & 6 & 3,0 \\
\hline Grupo orientação hipertensão & 1 & 1,0 & 4 & 3,5 & 5 & 2,0 \\
\hline Grupo de Lian Gong* & 3 & 3,0 & - & - & 3 & 1,0 \\
\hline Grupo de caminhada* & 3 & 3,0 & - & - & 3 & 1,0 \\
\hline
\end{tabular}

${ }^{*} p<0,05$ 
Em relação ao tempo de seguimento verificou-se que a média dos meses de atendimento do PSF foi significativamente menor $(\mathrm{p}<0,05)$ do que no programa tradicional ( $6 \pm 4$ vs $9 \pm 4)$. Os valores da pressão arterial dos hipertensos do PSF apresentaram queda significativa $(\mathrm{p}<0.05)$ entre o início e o final do período de 15 meses em que foi realizado o estudo $(152 \pm 24 / 92 \pm 15 \mathrm{~mm} \mathrm{Hg}$ vs $142 \pm 21 / 85 \pm 13 \mathrm{~mm} \mathrm{Hg}$ ), o que não ocorreu nos grupo seguido pelo programa tradicional. Por outro lado, quando comparou-se os valores da pressão arterial entre os grupos de pacientes estudados observou-se que a pressão sistólica inicial dos pacientes do PSF foi significativamente maior do que nos hipertensos do programa tradicional $(152 \pm 24$ vs $145 \pm 21 \mathrm{~mm} \mathrm{Hg})$. Porém, os valores da pressão diastólica final dos pacientes do grupo do PSF em relação aos hipertensos seguidos pelo sistema tradicional foram significativamente menores (85 \pm 13 vs $90 \pm 10 \mathrm{~mm} \mathrm{Hg}$ ). (Tabela 4)

Tabela 4 - Pressão arterial inicial e final (média \pm desvio padrão) e tempo de atendimento no Programa Saúde da Família e no Programa Tradicional (São Paulo, 2004)

\begin{tabular}{|c|c|c|}
\hline \multirow[b]{2}{*}{ Pressão Sistólica/Diastólica } & $\begin{array}{c}\text { Programa Saúde da } \\
\text { Familia }\end{array}$ & $\begin{array}{l}\text { Programa } \\
\text { Tradicional }\end{array}$ \\
\hline & $\%$ & $\%$ \\
\hline & 87 & 105 \\
\hline Pressão sistólica / diastólica inicial & $152 \pm 24^{\alpha *} / 92 \pm 15^{*}$ & $145 \pm 21 / 93 \pm 15$ \\
\hline Pressão sistólica / diastólica final & $142 \pm 21 / 85 \pm 13$ & $141 \pm 18 / 90 \pm 10^{\beta}$ \\
\hline Meses de atendimento & $6 \pm 4$ & $9 \pm 4$ \\
\hline
\end{tabular}

O controle da pressão arterial foi apenas de $20 \%$ nos hipertensos do PSF e $30 \%$ nos hipertensos do grupo tradicional. Acrescenta-se ainda, que os hipertensos não controlados foram significativamente diferentes $(\mathrm{p}<0,05)$ dos pacientes controlados nos seguintes as- pectos: menor renda salarial (44\% vs 20\%), maior tempo de diagnóstico de hipertensão ( $18 \%$ vs $9 \%$ com mais de 6 anos), doenças associadas (4\% vs $2 \%$ ) e para o tipo de consulta, mais situações de pronto atendimento $(51 \%$ vs 34\%). (Tabela 5)

Tabela 5 - Controle da pressão arterial dos hipertensos atendidos no Programa Saúde da Família e no Programa Tradicional (São Paulo, 2004)

\begin{tabular}{lcccccc}
\hline & \multicolumn{2}{c}{$\begin{array}{c}\text { Programa Saúde } \\
\text { da Familia }\end{array}$} & \multicolumn{2}{c}{ Programa } & \multicolumn{2}{c}{ Total } \\
Controle & $\mathbf{N}$ & $\mathbf{\%}$ & $\mathbf{N}$ & $\%$ & $\mathbf{N}$ & $\%$ \\
\hline Controlado & 26 & 20,0 & 21 & 30,0 & 47 & 25,0 \\
Não controlado & 61 & 80,0 & 84 & 70,0 & 145 & 75,0 \\
Total & $\mathbf{8 7}$ & $\mathbf{1 0 0}$ & $\mathbf{1 0 5}$ & $\mathbf{1 0 0}$ & $\mathbf{1 9 2}$ & $\mathbf{1 0 0}$ \\
\hline
\end{tabular}

\section{DISCUSSÃO}

Os dados do presente estudo evidenciaram que os hipertensos do PSF se diferiram dos pacientes seguidos pelo sistema tradicional quanto a variáveis biossociais. Em relação a etnia houve predomínio de brancos e essa variável deve ser considerada pois estudo mostrou que o controle em hipertensos negros e mulatos na cidade de Salvador-BA, foi menor que nos brancos ${ }^{(11)}$. A idade mais elevada merece atenção. Tem-se verificado o benefício extraordinário do tratamento anti-hipertensivo em idosos e reforça-se a influência da idade na gênese da hipertensão $0^{(12-13)}$. A baixa escolaridade pode ser um fator dificultador para melhorar a adesão ao tratamento, além de que investigações evidenciaram que a baixa escolaridade se associa com hipertensão arterial ${ }^{(14)}$.
Em relação aos hábitos de vida, os hipertensos do programa tradicional realizavam mais atividade física que os do PSF e é inegável a eficácia da atividade física regular na redução dos níveis da pressão arterial ${ }^{(15)}$. No tocante a outros hábitos de vida verificou-se que os hipertensos do PSF eram menos tabagistas e ingeriam mais bebida alcoólica.O uso abusivo de álcool relacionase a maior mortalidade cardiovascular. Além disso o álcool pode causar atenuação da potência anti-hipertensiva das drogas tendo seu efeito revertido sobre a pressão arterial somente duas semanas após a redução do hábito de beber ${ }^{(16)}$. Segundo orientações das IV Diretrizes ${ }^{(1)}$ deve-se limitar a ingestão a $30 \mathrm{ml} /$ dia de etanol para homens e $15 \mathrm{ml} / \mathrm{dia}$ para as mulheres, o que corresponde a $720 \mathrm{ml}$ de cerveja, $240 \mathrm{ml}$ de vinho e $60 \mathrm{ml}$ de bebida destilada. 
A importância de se modificar os hábitos de vida de uma população através de grupos educativos que forneçam informação e instrumentos para esta melhora é parte dos objetivos de alguns programas de saúde. Estes grupos têm sido trabalhados dentro do PSF através de união com a comunidade e a equipe de saúde.

Adiciona-se a esse contexto o fato de que os hipertensos atendidos pelo PSF apresentaram mais outras doenças, além da hipertensão e níveis de triglicérides mais elevados que também podem influenciar a adesão pelas dificuldades encontradas na realização de múltiplos tratamentos.

$\mathrm{Na}$ busca de uma melhor avaliação do controle da pressão arterial, foram analisadas as pressões iniciais e finais, no período total de estudo. Apesar dos pacientes hipertensos do PSF apresentarem apenas $20 \%$ de controle, comparando-se os dois grupos de hipertensos observou-se que apenas os hipertensos do PSF apresentaram redução significativa da pressão arterial e em menor tempo de tratamento. Ainda assim os hipertensos de ambos os grupos estão muito aquém do desejável controle da pressão arterial. A falta de controle adequado dos níveis da pressão arterial dos hipertensos é um fato. No Brasil não há levantamentos nacionais que informem a quantidade exata de hipertensos controlados. Estudos isolados ${ }^{(8,16)}$ têm mostrado baixos índices de controle, como o realizado na cidade de São Paulo, no qual a taxa de controle pressórico foi de 20,9\% para os hipertensos e $23,4 \%$ para os hipertensos diabéticos ${ }^{(16)}$.

O controle da pressão arterial e a adesão ao tratamento estão intimamente relacionados, os fatores que interferem na adesão nem sempre são fáceis de detectar e para melhorar o controle da hipertensão arterial é importante identificar aqueles pacientes que não aderem ao tratamento. Adesão é um processo complexo e um desafio que envolve a atuação tanto dos pacientes como dos profissionais de saúde. Os fatores que interferem na adesão ao tratamento anti-hipertensivo, podem ser relacionados ao paciente: como sexo, idade, raça, escolaridade, etnia, estado civil, ocupação, nível sócio econômico e religião. Outros relacionados com a própria doença, como a cronicidade, conhecimento sobre a patologia, e complicações, além dos aspectos culturais, crenças, perspectivas do tratamento e envolvimento familiar devem ser avaliados. O modo como a equipe de saúde relaciona-se com os pacientes, o vínculo estabelecido, além de especificidades do tratamento também influenciam no processo de adesão ${ }^{(17)}$.

O controle da hipertensão, a manutenção de níveis tensionais, exige intervenção constante e programada aos pacientes, incentivando a mudança de estilos de vida e mantendo de modo adequado o tratamento ${ }^{(17)}$.

O Programa Saúde da Família tem em seu funcionamento a atuação multidisciplinar, e pode-se ressaltar a atuação do enfermeiro como um fator a mais para facilitar a adesão do paciente ao tratamento e do controle da hipertensão como de outras doenças crônicas.

O presente estudo mostrou que houve diferença significativa nos tipos de atendimento, como a consulta com enfermeira, visita domiciliar médica e visita domiciliar de enfermeira que foram mais freqüentes para os hipertensos do PSF, por se tratar de formas exclusivas de consulta do programa. Em relação aos grupos oferecidos, em espaços cedidos pela comunidade, como o Lian Gong (ginástica oriental), grupo de qualidade de vida com orientações sobre saúde e caminhada, apesar de pouco freqüentes, somente os pacientes do PSF tiveram essas formas de participação. Pode-se atribuir esse fato ao local onde são realizados os grupos e através da divulgação e vínculo dos agentes comunitários com os pacientes.

\section{CONCLUSÕES}

O presente estudo evidenciou diferenças entre os hipertensos do grupo do PSF e do programa tradicional relativas a fatores biossociais, características da doença, hábitos de vida, exames laboratoriais, doenças associadas e tipos de atendimentos. Entretanto, a pressão arterial quando comparada à média de tempo em relação às reduções de níveis pressóricos significativas entre as pressões iniciais e finais e entre os grupos observou-se que ambos os grupos apresentam redução entre as pressões iniciais e finais, porém, os hipertensos do PSF foram os únicos em que a redução foi significativa (152 \pm $24 / 92 \pm 15$ vs $142 \pm 21 / 85 \pm 13 \mathrm{~mm} \mathrm{Hg}$ ) e em menor tempo que os pacientes do programa tradicional. Essas conclusões apontam para a necessidade de melhor integração multidisciplinar, melhora na organização das funções e atividades dos profissionais do PSF com o programa tradicional, e a promoção de atividades com os hipertensos para promover uma melhor adesão ao tratamento, melhorando o controle da hipertensão, prevenindo complicações que comprometem a saúde dos hipertensos.

\section{REFERÊNCIAS}

1. Sociedade Brasileira de Hipertensão. Sociedade Brasileira de Cardiologia. Sociedade Brasileira de Nefrologia. Diretrizes de hipertensão arterial, 4. Campos do Jordão; 2002.

2. Lólio CA. Prevalência de hipertensão arterial em Araraquara. Arq Bras Cardiol. 1990;55(3):167-73.

3. Freitas OC, Carvalho FR, Neves JM, Veludo PK, Parreira RS, Gonçalves RM et al. Prevalência de hipertensão arterial sistêmica na população urbana de Catanduva, SP. Arq Bras Cardiol. 2001; 77(1):9-15. 
4. The Seventh Report of the Joint National Committee on Prevention, Detection, Evaluation, and Treatment of High Blood Pressure. The JNC 7 report. JAMA. 2003;289(19):2560-72.

5. 2003 European Society of Hypertension. European Society of Cardiology guidelines for the management of arterial hypertension. J Hypertens. 2003;21(6):1011-53

6. Sanchez CG, Pierin AMG. Perfil do paciente hipertenso atendido em pronto socorro: comparação com hipertensos em tratamento ambulatorial. Rev Esc Enferm USP. 2004;38(1):90-8.

7. Strelec MAAM, Pierin AMG, Mion Júnior D. A influência do conhecimento sobre a doença e a atitude frente à tomada de remédios no controle da hipertensão arterial. Arq Bras Cardiol. 2003;81(4):349-54.

8. Ministério da Saúde. Instituto para Desenvolvimento da Saúde (IDS). Programa Saúde da Família. Manual de enfermagem. Brasília (DF); 2001.

9. Pierin AMG, Mion Júnior D. Atuação da equipe de enfermagem na hipertensão arterial. In: Ministério da Saúde. Instituto para o Desenvolvimento da Saúde (IDS). Programa Saúde da Família. Manual de enfermagem. Brasília (DF); 2001; p.149-54.

10. Rosner B. Fundamentals of biostatistics. New York: Duxbury Press; 1994. p. 682.

11. Lessa I, Fonseca J. Raça. Aderência ao tratamento e/ou consultas e controle da hipertensão arterial. Arq Bras Cardiol. 1997; 68(6):443-9.
12. Cooperative Research Group. Final results of the Systolic Hypertension in the Elderly Program (SHEP). Prevention of stroke by antihypertensive drug treatment in older persons with isolated systolic hypertension. JAMA. 1991; 265(24):3255-64.

13. Cabral AH. Caracterização dos hipertensos atendidos nas Unidades Básicas de Saúde da cidade de Sorocaba. [tese]. São Paulo: Escola de Enfermagem da USP; 2000.

14. Bambirra AP, Assunção JH, Monteiro JM, Pierin AMG, Mion Junior D et al. Hypertension in employes of a University General Hospital. Rev Hosp Clin Un Med São Paulo. 2004; 59(6):329-36.

15. Trombetta IC. Excercício físico e dieta hipocalórica para o paciente obeso: vantagens e desvantagens. Rev Bras Hipertens. 2003; 109(1):130-3.

16. Pierin AMG, Mion Junior D, Fukushima JT, Pinto A, Kaminaga M. O perfil de um grupo de pessoas hipertensas de acordo com o conhecimento e gravidade da doença. Rev Esc Enferm USP. 2001;35(1):11-8.

17. Pierin AMG, Strelec MAAM, Mion Junior D. O desafio do controle da hipertensão arterial e a adesão ao tratamento. In: Pierin AMG, coordenadora. Hipertensão arterial: uma proposta para o cuidar. Barueri (SP): Manole; 2004. cap. 16, p. 275-89. 